\title{
Approche SIG Pour La Modélisation Du Réseau Routier Et La Mesure De L'accessibilité Aux Équipements Publics. Cas De La Ville d'Agadir
}

\author{
Charaf eddine Benichou, Doctorant
}

L.E.G.A.C, Faculté des Lettres et des Sciences Humaines, Sais Fès, Université Sidi Mohamed Ben Abdellah, Fès, Maroc

\author{
Mokhlis Derkaoui Alaoui, PhD
}

Faculté des Lettres et des Sciences Humaines

Université Ibn Zohr, Agadir, Maroc

\section{Ali Faleh, PhD}

L.E.G.A.C, Faculté des Lettres et des Sciences Humaines, Sais Fès Université Sidi Mohamed Ben Abdellah, Fès, Maroc

\section{Doi: 10.19044/esj.2018.v14n2p38 URL:http://dx.doi.org/10.19044/esj.2018.v14n2p38}

\begin{abstract}
The study of road networks and accessibility to public facilities in Morocco doesn't receive enough importance in regional planning. Most of the work carried out in the field is limited to diagnosis, relying on traditional tools of analysis and description. The goal of this article is to propose a thorough analysis of public facilities accessibility using isochronous maps, which will not only make it possible to evaluate the quality of urban transport system, but also guarantee the equity of the geographical distribution of public services. The study area is the urban commune of Agadir city on Morocco's southwest coast. The approach used for this purpose is based on urban road network modeling, travel time calculations and geographic information software (GIS). Therefore, a Geographical Information System dedicated to Transport (GIST) was built, supplied with structured data in a geodatabase, and analyzed by using ArcGIS Network Analyst extension. The results demonstrate that Access to public facilities in the city of Agadir differs in quality according to districts and the types of services. However, the most remote marginal areas remain the most affected areas, both in terms of equipment distribution. the availability of transport infrastructure, or the quality of service in urban public transport.
\end{abstract}

Keywords: GIS, accessibility, road network, urban transport, Agadir 


\section{Résumé}

L'étude des réseaux routiers et de l'accessibilité aux équipements publics au Maroc, n'a pas requis assez d'importance en aménagement du territoire, alors que la majorité des travaux réalisés en la matière ne dépassent pas l'état de diagnostic, via les outils traditionnels d'analyse et de description. L'objectif de cette étude est d'analyser l'accessibilité aux équipements publics, en la représentant sous forme de cartes isochrones, ce qui permettra d'évaluer la qualité du système de transport urbain, ainsi que l'équité de la répartition géographique des services publics. La zone d'étude est la commune urbaine de la ville d'Agadir au sud-ouest du Maroc.

L'approche utilisée à cet effet est basée sur la modélisation du réseau routier urbain, des calculs de temps de déplacement et des fonctions intégrées dans les logiciels d'informations géographiques (SIG). Ainsi, un Système d'Informations Géographiques dédiés au Transport (SIG-T) a été construit, alimenté de données structurées dans une geodatabase, puis analysé en utilisant 1'extension ArcGIS Network Analyst.

Les résultats montrent que la qualité d'accès aux équipements publics à la ville d'Agadir diffère selon les quartiers et les types de services, cependant, les quartiers marginaux les plus éloignés demeurent les zones les plus touchés, tant en termes de répartition des équipements publics, de la disponibilité des infrastructures de transport, ou de la qualité de desserte en transport public urbain.

Mots-clés : SIG, accessibilité, réseau routier, transport urbain, Agadir

\section{Introduction}

La géographie des transports repose sur une multitude de points de vue qui résultent de la complexité et du degré d'importance attribués au transport dans la dynamique des territoires. Le système de transport est une des solutions que la société se donne pour maîtriser les discontinuités spatiales. Il groupe les lieux en une structure cohérente d'organisation spatiale et influence la localisation des agents.

La présente étude du réseau routier urbain et de l'accessibilité aux équipements collectifs, n'essaie pas de se démarquer des études déjà réalisés, mais plutôt d'aborder un autre aspect de l'analyse des transports urbains : la modélisation. Modélisation qui ne serait possible qu'avec l'avènement de l'outil informatique en général et des Systèmes d'Informations Géographiques (SIG) en particulier.

Les résultats sont représentés sous forme de cartes d'accessibilité, leur importance vient du fait qu'elles permettent d'évaluer, avec une grande précision, le niveau de la justice socio-spatiale à la zone d'étude qu'est la ville 
d’Agadir, révélant ainsi les zones mal équipées ou mal desservies par le transport public urbain public.

\section{Problématique}

Comme tout autre concept dans le domaine des sciences humaines, l'accessibilité a fait l'objet de nombreuses appréhensions au travers de différents points de vue.

De ces perspectives découlerons différentes approches et mesures de l'accessibilité, catégories spatiales de l'accessibilité et les problèmes de l'accessibilité (géographique).

L'accessibilité se définit comme étant « la capacité d'un endroit à être atteint à partir d'autres endroits de localisation géographique différente » (Rodrique, 2006). En géographie, l'accessibilité d'un lieu est généralement définie comme « la plus ou moins grande facilité avec laquelle ce lieu peut être atteint à partir d'un ou de plusieurs autres lieux, par un ou plusieurs individus susceptibles de se déplacer à l'aide de tout ou partie des moyens de transport existants ». (CERTU, 2006), mais la question qui se pose est comment mesurer l'accessibilité ? dans notre cas, comment mesurer l'accessibilité à un équipement public en milieu urbain?

En effet, toute étude qui s'intéresse à l'accessibilité et ne l'étudie qu'en utilisant la distance euclidienne entre les endroits objets de l'étude, risque de fausser les résultats, sinon de vider l'étude de son contenu. L'étude de l'accessibilité doit prendre en compte le support des déplacements, et qui n'est autre que le réseau de transport (Derkaoui, 2014).

\section{Méthodologie}

\section{Recensement des données}

Le recensement des données persistantes, ou vitales, ne peut se faire qu'en suivant une démarche systématique appuyant une réflexion rigoureuse à laquelle prend part non seulement le modélisateur mais aussi les utilisateurs ou les clients du système à réaliser. L'identification d'une donnée persistante est avant tout une affaire de jugement. Elle dépend fortement du contexte et du domaine où s'exerce le travail d'analyse des besoins par le modélisateur.

C'est alors qu'il a fallu d'abord recueillir les données nécessaires (réseau routier, réseau de transport, équipements publics) pour alimenter le système d'information géographique dédié au transport: SIG-T. La particularité des SIG-T tient au fait qu'ils intègrent des réseaux de transport et permettent ainsi de répondre à la question : « combien de temps ? » (Mercier, 2008).

Un travail de terrain a été essentiel, afin d'alimenter le SIG-T en données nécessaire pour l'analyse, les outils utilisés sont un GPS professionnel doté d'un SIG nomade pour relever les données relatives aux 
réseaux de transport par bus, et un chronomètre pour calculer les temps de déplacements via les différents modes de transport urbain.

Ce recensement des données persistantes à ce système va constituer la première étape vers la mise en place du système d'information géographique dédié au transport, ensuite une autre étape du processus de mise en point de ce système d'information prend place : modélisation des données, à savoir créer un Modèle conceptuel de données, Modèle logique de données et un modèle physique de données.

\section{Traitement des données}

Cette étape consiste à organiser les données recueillies et les structurer dans une base de données géographique, cette géodatabase fournit une structure pour les informations géographiques et prend en charge les classes d'entités topologiques intégrées et permet la modélisation des transports multimodale, à cette implémentation, seront ajoutées des précisions concernant les classes d'entités, les relations et la règle de topologie utilisée.

La structure de la géodatabase comme le montre la figure 1 sur laquelle se base notre travail de modélisation du réseau routier urbain et de l'accessibilité est une traduction du modèle de données réalisé.

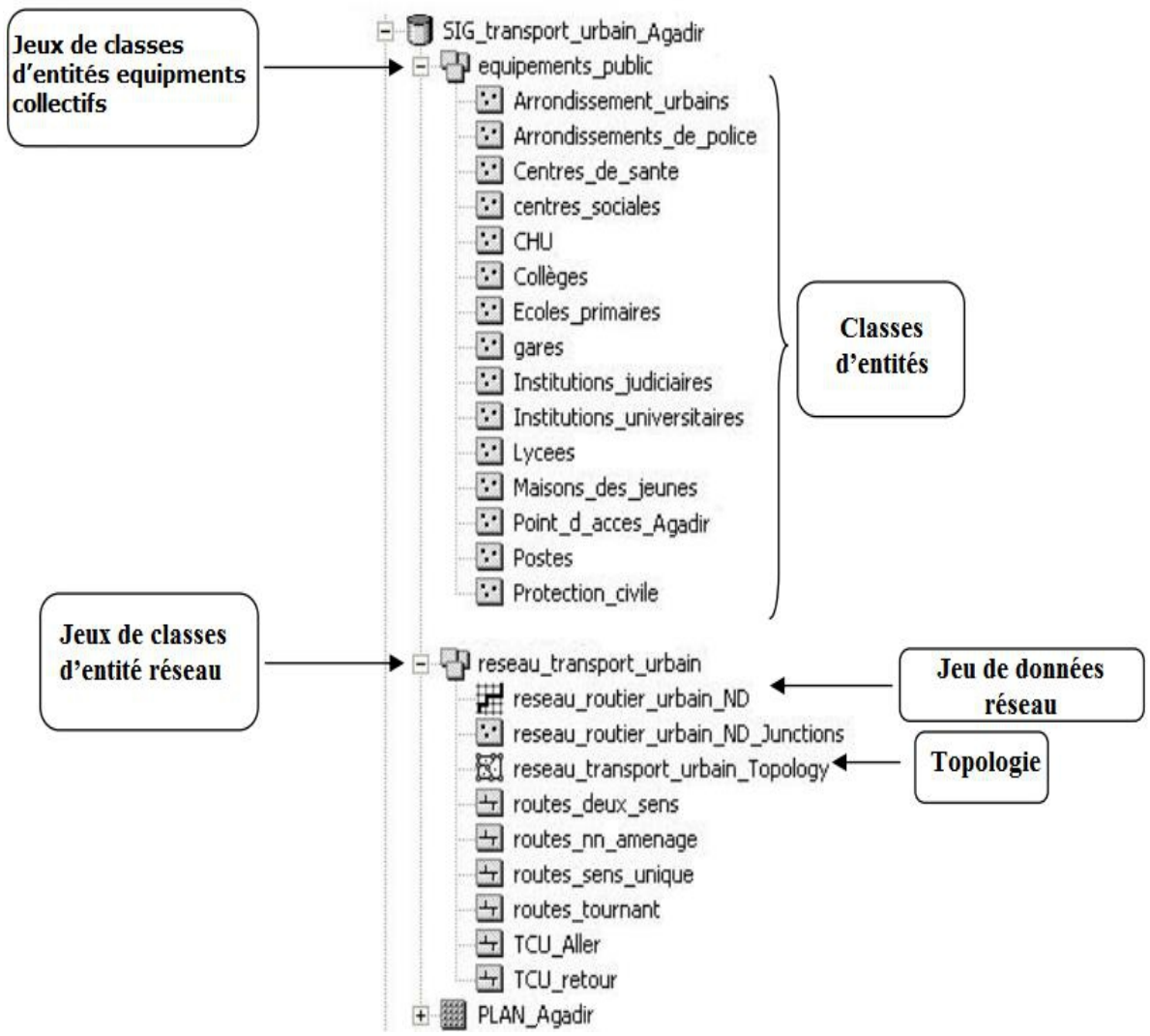

Figure. 1 La geodatabase 
Les jeux de données réseau sont constitués d'éléments de réseau. Les éléments de réseau sont générés à partir des sources utilisées pour créer le jeu de données réseau. La géométrie des entités source sert à établir la connectivité. De plus, les éléments de réseau (tronçons, jonctions et les tournants) possèdent des attributs qui gèrent la navigation sur le réseau.

\section{Outil d'analyse}

L'analyse du réseau routier urbain menée s'appuiera sur une analyse à partir de l'utilisation directe des données collectées, ainsi l'étude de l'accessibilité aux équipements collectifs, utilise les attributs du réseau routier pour analyser la situation de l'accessibilité d'un équipement ou d'un ensemble d'équipements présentant le caractère de rendre à la population urbaine le même service.

C'est donc une étape dans laquelle on a essayé d'exploiter tout le travail fait dans les étapes précédentes et d'analyser l'accessibilité aux équipements publics en utilisant l'extension ArcGis Network Analyst.

\section{Calcul et critères d'analyse}

En milieu urbain, on ne peut pas imaginer un calcul/une mesure de l'accessibilité sans un réseau routier urbain, qui servira de support pour cette mesure. Sans lui toute étude de l'accessibilité sera limitée au calcul des distances à vol d'oiseau, ce qui le videra de toute signification (Assioui et al, 2012).

L'espace-temps calculé en minutes est l'unité de mesure adopté pour évaluer l'accessibilité, un calcul basé uniquement sur la distance risque de fausser les résultats.

Le choix des critères d'analyse est influencé d'un côté, par les normes éditées par le ministère de l'habitat et de l'urbanisme (2005), et d'un autre côté, par l'analyste, qui par son sens critique évalue, repense et choisi les critères adéquats et qui varieront selon l'équipement public choisi et sa localisation sur le territoire.

\section{Les principaux apports et résultats}

Ce travail essaie de donner une vision globale sur la structure et les caractéristiques du réseau routier urbain et l'accessibilité aux équipements collectifs au sein de l'espace urbain de la ville d'Agadir. Vision qui prétend dépasser les analyses classiques basées sur l'illustration des chiffres sans tenir compte de l'aspect spatial du phénomène étudié, le premier résultat est la base de ce travail, il représente la modélisation du réseau routier urbain d'Agadir : Dans cette optique, l'analyse de l'accessibilité aux équipements collectifs est influencée directement par les choix de l'analyste. Les choix des critères d'analyse se varieront selon le type de l'équipement collectif choisi, sa 
localisation dans l'espace urbain et le nombre des autres équipements collectifs offrant le même service à la population.

Ci-après des exemples des cartes générées représentant l'accessibilité aux différents équipements publics :

\section{L'accessibilité piétonne aux écoles primaires (figure 3)}

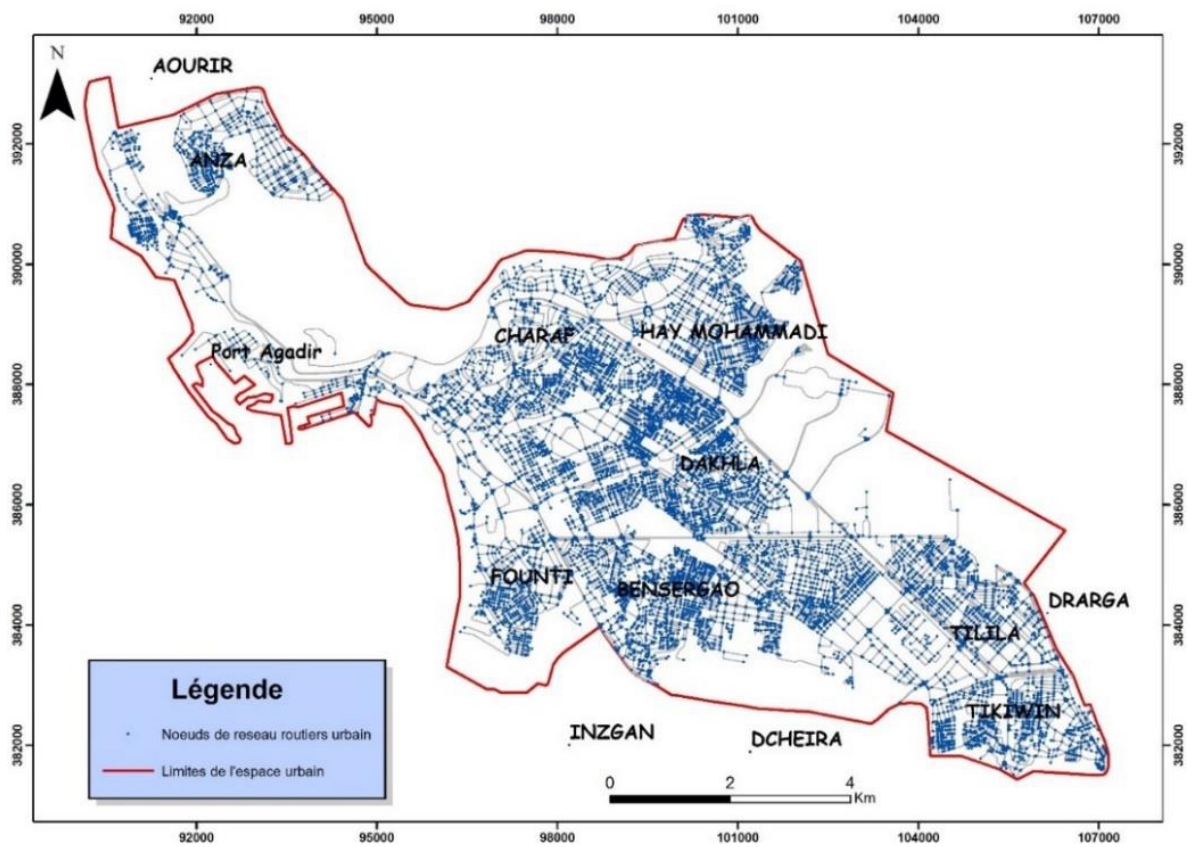

Figure. 2 : La carte du réseau routier urbain modélisé

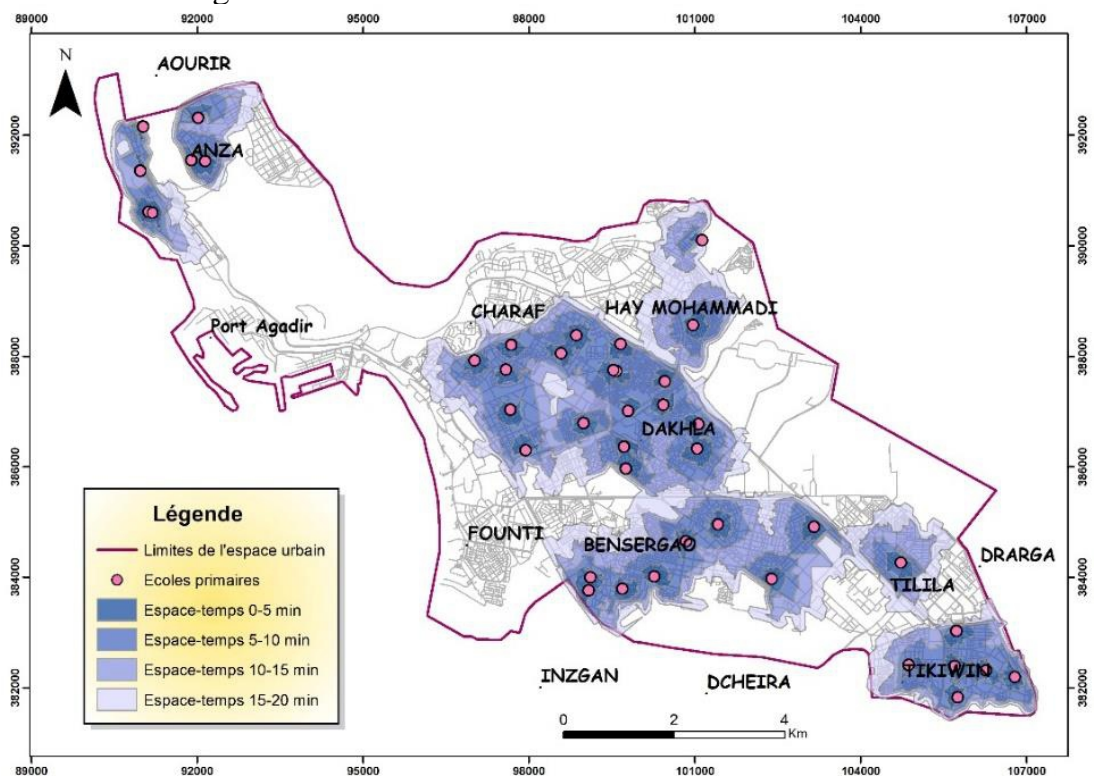

Figure.3 : Carte de l'accessibilité piétonne aux écoles primaires 
L'accessibilité piétonne aux écoles primaires au sein de l'espace urbain de la ville d'Agadir est généralement jugée bonne. Les zones d'une accessibilité de plus de 20 min en espace-temps sont, en fait, soit des quartiers nouvellement construits (l'est d'Anza et de Tilila), soit des quartiers bourgeois où la scolarité des enfants se fait dans des écoles du secteur privé, soit des quartiers des zones industrielles ou touristiques.

\section{- L'accessibilité bimodale (BUS et piétonne) aux établissements universitaires (figure 4)}

La carte de l'accessibilité bimodale (BUS et piétonne) aux établissements universitaires montre que la desserte en bus de certains quartiers de la ville n'est pas en encore satisfaisante, surtout les quartiers du nord de la ville d'Agadir, l'espace-temps dépasse 30min, et cela même en heure creuse. Les étudiants qui habitent dans les communes avoisinantes ont besoin de beaucoup plus de temps pour arriver à leurs établissements universitaires, ils sont dans plusieurs cas, obligés d'utiliser deux bus, ce qui rend leurs déplacements difficiles.

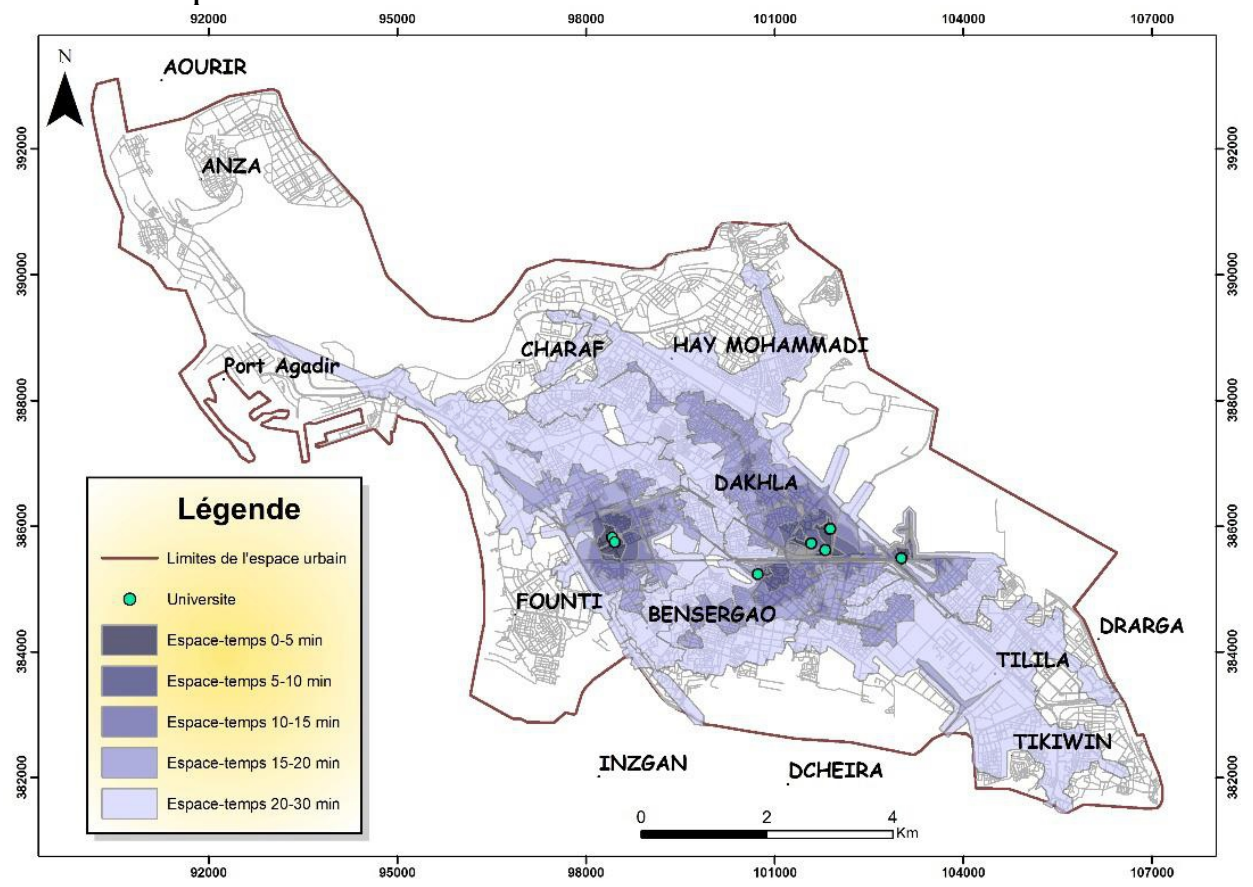

Figure. 4 : La carte l'accessibilité bimodale (BUS et piétonne) aux établissements universitaires en heure creuse

- Accessibilité automobile à la caserne des sapeurs-pompiers en heure pointe et en heure creuse (figure 5)

L'accessibilité des sapeurs-pompiers aux différents composantes de la ville est une question vitale, par contre, l'observation des deux cartes représentant l'accessibilité isochrone des sapeurs-pompiers, montre que 
l'accès aux quartiers nord d'Agadir ainsi que le port n'est pas assez bonne, l'espace-temps d'accès dépasse largement les $15 \mathrm{~min}$, d'où la nécessité d'implanter une caserne de pompiers au nord de la ville.
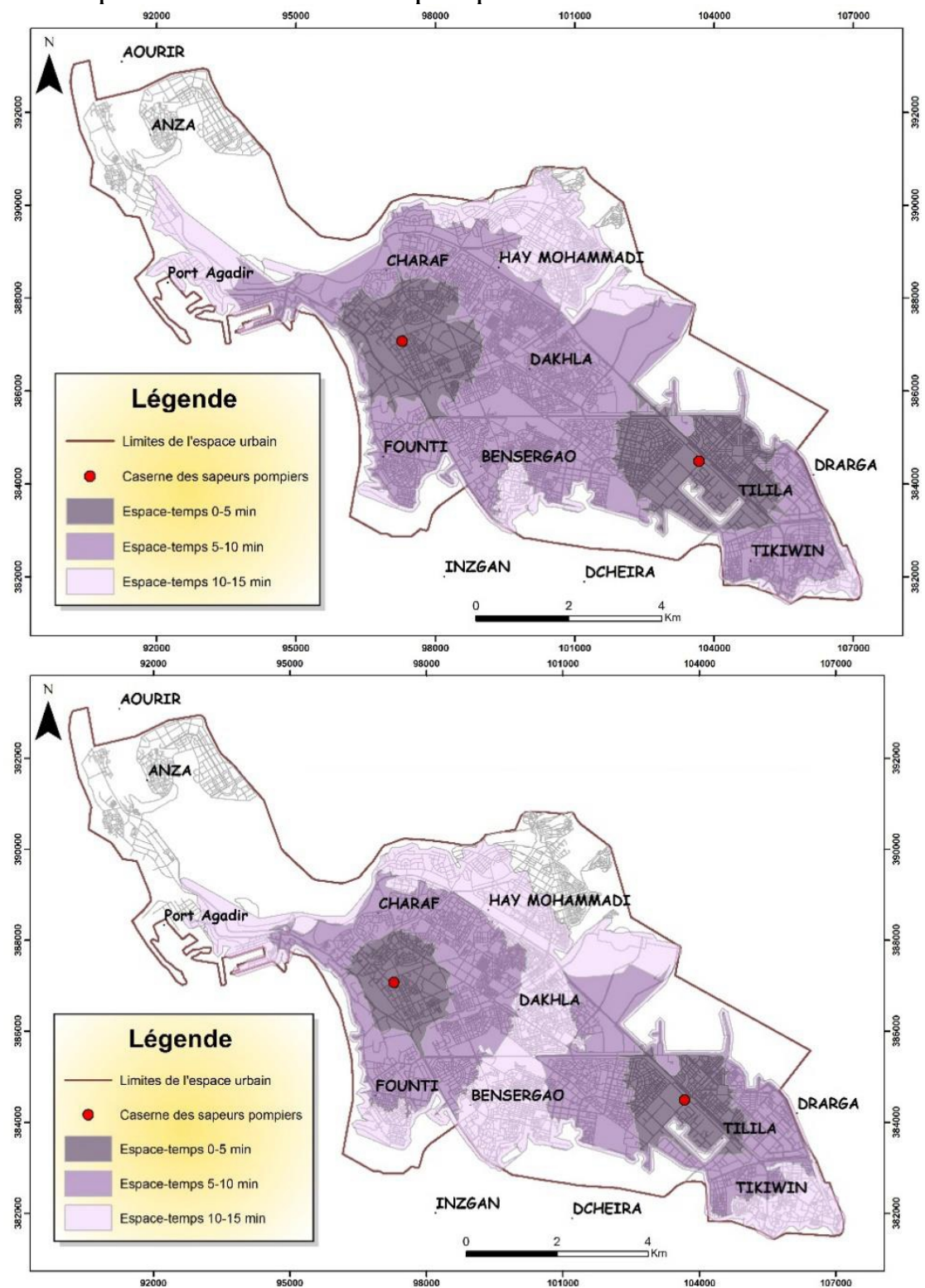

Figure. 5 : Les cartes de l'accessibilité automobile aux casernes des sapeurs-pompiers en heure pointe et en heure creuse

Les résultats obtenus seront considérés comme un outil d'aide à la prise de décision pour les collectivités locales et les décideurs publics. Cet outil peut servir aussi à développer des applications dans le secteur privé, que ce soit 
pour la gestion de son parc automobile ou l'étude de leurs installations ainsi que le niveau de leur accessibilité.

\section{Conclusion}

Ce travail s'inscrit dans une nouvelle approche de l'étude des réseaux de transport et de l'accessibilité. Approche basée sur l'utilisation des nouvelles technologies de l'information comme outil d'aide à l'analyse.

Ce choix trouve sa justification dans le fait que la masse des données à analyser et les traitements nécessaires rendent le recours à ces outils indispensable.

L'absence de toute étude antérieure de modélisation du réseau de transport que ce soit pour la ville d'Agadir ou toute autre ville marocaine, qui pourrait nous guider à travers leur résultats et limites, a beaucoup pesé sur notre étude de modélisation dans le sens où on était mené à donner.

La qualité d'accès aux équipements publics à la ville d'Agadir diffère selon les quartiers et les types de services, cependant, les quartiers marginaux les plus éloignés demeurent les zones les plus touchés, tant en termes de répartition des équipements publics, de la disponibilité des infrastructures de transport, ou de la qualité de desserte en transport public urbain.

L'étude de l'accessibilité nécessite de prendre en considération ses dimensions économiques, sociales et culturelles, ainsi que l'influence d'autres facteurs tels que le revenu, l'âge et le sexe. L'évaluation des répercussions de ces variables sur la mobilité quotidienne des différentes catégories de la population est très prometteuse.

\section{References:}

1. Assioui,M. Essadek, A. Lahrar, A. (2012). L'accessibilité aux équipements collectifs en milieu urbain, un essai d'analyse et d'évaluation, cas de la ville d'Oujdà. Colloque international des utilisateurs de SIG, Taza GIS days 23-24 Mai, recueil des résumés, Université Sidi Mohamed Ben Abdellah, Fès.

2. Mercier, A. (2008). Accessibilité et évaluation des politiques de transport en milieu urbain : le cas du tramway strasbourgeois. Thèse de doctorat en sciences économiques, Université de Lumière Lyon 2.

3. CERTU (2006). Calculs d'accessibilité, impacts des spécifications du réseau routier sur les calculs d'accessibilité, données sources méthodes.

4. Derkaoui Alaoui, M. (2014). Transport à la ville de Fès, étude sur les déplacements urbains et l'accessibilité aux équipements publics en utilisant les Systèmes d'Informations Géographiques, thèse de doctorat en géographie, Faculté des Lettres et des Sciences Humaines, Sais-Fès. 
5. Ministère délégué chargé de l'habitat et de l'urbanisme, Direction de l'urbanisme. (2005): Normes urbaines des équipements collectifs de proximité.

6. Rodrigue, J.P. (2006). The Geography of transport systems. Routledge, Abingdon. 